\title{
ACTIONS DE GROUPES DE KAZHDAN SUR LE CERCLE
}

\author{
PAR ANDRÉS NAVAS
}

RÉSUMÉ. - Nous démontrons que tout homomorphisme d'un groupe de Kazhdan discret dans le groupe des difféomorphismes directs du cercle de classe $C^{1+\alpha}(\alpha>1 / 2)$ a une image finie.

(c) 2002 Éditions scientifiques et médicales Elsevier SAS

ABSTRACT. - We prove that every homomorphism from a discrete Kazhdan group to the group of orientation-preserving diffeomorphisms of the circle of class $C^{1+\alpha}(\alpha>1 / 2)$ has a finite image.

(c) 2002 Éditions scientifiques et médicales Elsevier SAS

\section{Introduction}

On a l'impression générale que les groupes qui ont la propriété $(\mathrm{T})$ de Kazhdan ne peuvent pas agir de manière non triviale sur des espaces munis d'une certaine structure unidimensionnelle. Par exemple, on sait que toute action par isométries d'un tel groupe sur un arbre réel a un point fixe global (voir [1] et [19]).

Pour des actions par homéomorphismes de la droite, on a quelques résultats (voir [20]). Néanmoins, le problème principal dans ce cas, celui de savoir si un groupe de Kazhdan peut agir de manière fidèle par homéomorphismes de la droite, reste encore ouvert, même pour certains réseaux dans des groupes de Lie simples de rang réel $\geqslant 2$ (ces groupes ont la propriété $(\mathrm{T})$ ). Remarquons que pour le cas des groupes discrets dénombrables, ce problème est équivalent au problème de savoir si le groupe admet un ordre total invariant à gauche (voir [9]).

Pour des actions sur le cercle, on dispose de résultats plus précis. Notamment, dans [8], É. Ghys démontre que pour toute représentation $\phi: \Gamma \rightarrow \operatorname{Diff}_{+}^{1}\left(\mathrm{~S}^{1}\right)$ d'un réseau $\Gamma$ d'un groupe de Lie simple de rang réel $\geqslant 2$, l'image $\phi(\Gamma)$ est finie. Dans [3], M. Burger et N. Monod obtiennent le même résultat pour une famille un peu différente de réseaux. Ce résultat a été récemment amélioré dans [4]. Dans [20] et [21], le lecteur trouvera des résultats analogues obtenus à partir de la relation entre le problème d'ordonabilité d'un groupe et ses actions sur la droite et le cercle. Voir aussi [6] pour le cas analytique.

Dans cet article, nous étendons ces résultats aux groupes de Kazhdan sous une hypothèse de régularité un peu plus forte. Plus précisément, nous démontrons le théorème suivant.

THÉORÈME. - Soient $\Gamma$ un groupe discret et $\phi: \Gamma \rightarrow \operatorname{Diff}_{+}^{1+\alpha}\left(\mathrm{S}^{1}\right)$ une représentation, avec $\alpha>1 / 2$. Si $\Gamma$ possède la propriété $(\mathrm{T})$ de Kazhdan, alors l'image $\phi(\Gamma)$ est finie.

La preuve de ce théorème est fortement inspirée par le travail [17] de A. Reznikov dans lequel ce dernier considère un cocycle introduit par G. Segal (voir [16]) et obtient quelques résultats partiels (toujours sous les mêmes hypothèses de régularité). Par exemple, A. Reznikov démontre que la représentation unitaire de $\Gamma$ sur $\mathcal{L}_{\mathbb{C}}^{2}\left(\mathrm{~S}^{1}\right)$ par demi-densités est réductible, et il montre 
aussi que $\Gamma$ ne contient pas d'élément conjugué dans $\operatorname{Diff}_{+}^{1+\alpha}\left(\mathrm{S}^{1}\right)$ à un élément parabolique ou hyperbolique de $\operatorname{PSL}(2, \mathbb{R})$. Nous utilisons aussi le cocycle introduit dans [16] et [17], mais notre approche au problème est en général plus géométrique.

Comme corollaire on obtient le résultat suivant, qui avait été déjà démontré par A. Reznikov dans [17], et qui répond à une question posée par É. Ghys et V. Sergiescu dans [11], p. 191, et par P. de la Harpe et A. Valette dans [14], p. 133.

COROLlaire. - Le groupe G de Thompson n'a pas la propriété (T).

Comme l'a défini Thompson, G est un sous-groupe du groupe des homéomorphismes directs et affines par morceaux du cercle. Néanmoins, dans [11] on démontre qu'il se plonge dans le groupe des difféomorphismes directs et de classe $C^{\infty}$ du cercle. Puisque $\mathrm{G}$ est un groupe discret non fini, on peut appliquer notre théorème pour obtenir le corollaire.

Le plan de cet article est le suivant.

Dans le $§ 1$, nous fixons les notations et nous faisons un rappel sur la propriété (T) de Kazhdan. Pour plus d'informations sur le sujet, nous renvoyons le lecteur intéressé à [14].

Dans le §2, nous introduisons le cocycle de Segal-Reznikov. Nous démontrons, à l'aide de ce cocycle, que sous les hypothèses de notre théorème, il existe un courant géodésique invariant. De plus, nous démontrons que ce courant satisfait certaines "propriétés de stabilité" qui seront importantes dans la suite.

Dans le $\S 3$ nous démontrons que sous nos hypothèses, l'action de $\phi(\Gamma)$ sur le cercle est libre. Nous finissons la preuve du théorème en utilisant un ancien résultat de Hölder concernant la commutativité des groupes qui agissent librement sur le cercle.

Dans le $\S 4$ nous étudions le cas non discret. Nous étendons notre théorème au cas d'un groupe localement compact séparable et à base dénombrable ayant la propriété (T).

Finalement, dans le $\$ 5$ nous donnons les idées d'une preuve alternative de notre résultat qui utilise le théorème de convergence de Tukia, Hinkkanen, Casson-Jungreis et Gabai.

\section{Notations et définitions}

\subsection{Le groupe $\operatorname{Diff}_{+}^{1+\alpha}\left(\mathrm{S}^{1}\right)$}

On considère le cercle unité $S^{1}$ muni de l'orientation canonique. On notera $(a, b)$ l'intervalle de $a$ à $b$ avec cette orientation. Les intervalles $(a, b],[a, b)$ et $[a, b]$ sont définis de manière analogue. Notons que si $b \in(a, c)$ alors $c \in(b, a)$ et $a \in(c, b)$. Nous noterons ces relations simplement par $a<b<c<a$. La distance entre $a$ et $b$ est la plus petite des longueurs de $(a, b)$ et $(b, a)$. Nous noterons parfois cette distance $|a-b|$.

Le revêtement universel de $\mathrm{S}^{1}$ est la droite $\mathbb{R}$ par l'application $x \mapsto \mathrm{e}^{\mathrm{i} x}$. On notera Homéo $_{+}\left(\mathrm{S}^{1}\right)$ le groupe des homéomorphismes directs du cercle, c'est-à-dire le groupe des homéomorphismes du cercle qui préservent l'orientation. Par abus de notation, pour chaque $f \in \mathrm{Homéo}_{+}\left(\mathrm{S}^{1}\right)$ nous noterons aussi $f$ l'un quelconque de ses relèvements à $\mathbb{R}$. Ainsi, $f: \mathbb{R} \rightarrow \mathbb{R}$ est une application strictement croissante vérifiant l'égalité $f(x+2 \pi)=f(x)+2 \pi$ pour tout $x \in \mathbb{R}$.

Nous noterons $I d$ l'application identité du cercle. Nous considérerons le groupe Diff ${ }_{+}^{1+\alpha}\left(\mathrm{S}^{1}\right)$ des difféomorphismes directs du cercle qui ont une dérivée Hölder continue d'exposant $\alpha \geqslant 0$, avec un inverse satisfaisant la même propriété. Nous noterons $\left|f^{\prime}\right|_{\alpha}$ la norme Hölder d'exposant $\alpha$ de la dérivée de $f$, c'est-à-dire,

$$
\left|f^{\prime}\right|_{\alpha}:=\sup _{x \neq y} \frac{\left|f^{\prime}(x)-f^{\prime}(y)\right|}{|x-y|^{\alpha}} .
$$




\subsection{Les propriétés $(\mathrm{T})$ de Kazhdan et $(\mathrm{FH})$ de Serre}

Nous n'aurons pas besoin de la définition classique de la propriété $(\mathrm{T})$. Nous utiliserons simplement le fait que tout groupe discret qui a cette propriété vérifie une autre propriété de rigidité introduite par J.-P. Serre et notée $(\mathrm{FH})$. En effet, un théorème de P. Delorme et A. Guichardet établit que ces deux propriétés sont équivalentes pour les groupes localement compacts séparables à base dénombrable (voir le Chapitre 5 de [14]).

Soit $\Gamma$ un groupe topologique quelconque et $\theta: \Gamma \rightarrow U(\mathcal{H})$ une représentation linéaire unitaire de $\Gamma$ sur un espace de Hilbert $\mathcal{H}$ (réel ou complexe). On dit que $\theta$ est continue si pour tout $\psi \in \mathcal{H}$, l'application $g \mapsto \theta(g) \psi$ est continue.

On dit que $c: \Gamma \rightarrow \mathcal{H}$ est un cocycle par rapport à $\theta$ si $c$ est une application continue et pour tout $g_{1}, g_{2} \in \Gamma$ on a $c\left(g_{2} g_{1}\right)=c\left(g_{1}\right)+\theta\left(g_{1}\right) c\left(g_{2}\right)$. On dit qu'un cocycle $c$ est un cobord s'il existe $\psi \in \mathcal{H}$ tel que $c(g)=\theta(g) \psi-\psi$ pour tout $g \in \Gamma$.

On note $Z^{1}(\Gamma, \theta)$ l'espace des cocycles et $B^{1}(\Gamma, \theta)$ le sous-espace des cobords. L'espace quotient $Z^{1}(\Gamma, \theta) / B^{1}(\Gamma, \theta)$ est appelé le premier espace de cohomologie de $\Gamma$ (à valeurs dans $\theta$ ) et noté $H^{1}(\Gamma, \theta)$.

DEFINITION 1.1. - Un groupe topologique $\Gamma$ possède la propriété $(\mathrm{FH})$ de Serre si pour toute représentation $\theta$ continue et unitaire de $\Gamma$, l'espace $H^{1}(\Gamma, \theta)$ est trivial.

Parmi les exemples de groupes localement compacts séparables à base dénombrable qui ont la propriété $(\mathrm{FH})$ (et donc la propriété $(\mathrm{T})$ ) on trouve les groupes compacts. On trouve aussi les groupes de Lie simples de rang réel $\geqslant 2$ et les groupes $S p(1, n), F_{4(-20)}$, ainsi que les réseaux dans ces groupes. D'autre part, dans [10], M. Gromov a construit une famille non dénombrable de groupes discrets qui ont la propriété (T). De plus, en suivant une idée de M. Gromov, dans [22] A. Zuk a démontré que "génériquement" tout groupe de présentation finie a la propriété de Kazhdan.

\section{Un courant géodésique invariant}

\subsection{Une représentation et un cocycle associé}

Dorénavant, et sauf dans le $\S 4, \Gamma$ désignera toujours un groupe discret.

Soit $\phi: \Gamma \rightarrow \operatorname{Diff}_{+}^{1+\alpha}\left(\mathrm{S}^{1}\right)$ une représentation de $\Gamma$ à valeurs dans le groupe des difféomorphismes directs du cercle de classe $C^{1+\alpha}, \alpha>1 / 2$. Pour simplifier la notation, nous noterons parfois le difféomorphisme $\phi\left(g^{-1}\right)$ simplement $g$.

On considère l'espace $\mathcal{H}:=\mathcal{L}_{\mathbb{R}}^{2, \Delta}\left(\mathrm{S}^{1} \times \mathrm{S}^{1}\right)$ des fonctions $K$ dans $\mathcal{L}_{\mathbb{R}}^{2}\left(\mathrm{~S}^{1} \times \mathrm{S}^{1}\right)$ antisymétriques, c'est-à-dire telles que $K(x, y)+K(y, x)=0$. On considère l'action $\theta$ de $\Gamma$ sur $\mathcal{L}_{\mathbb{R}}^{2, \Delta}\left(\mathrm{S}^{1} \times \mathrm{S}^{1}\right)$ donnée par

$$
(\theta(g) K)(r, s):=K(g(r), g(s)) \cdot\left[g^{\prime}(r) g^{\prime}(s)\right]^{1 / 2} .
$$

Il est facile de voir que $\theta$ est une représentation unitaire.

Pour chaque $g \in \Gamma$ considérons la fonction

$$
c(g)(r, s):=\frac{\left[g^{\prime}(r) g^{\prime}(s)\right]^{1 / 2}}{2 \tan \left(\frac{g(r)-g(s)}{2}\right)}-\frac{1}{2 \tan \left(\frac{r-s}{2}\right)} .
$$

On vérifie aisément la relation de cocycle $c\left(g_{2} g_{1}\right)=\theta\left(g_{1}\right) c\left(g_{2}\right)+c\left(g_{1}\right)$. En effet, $c(g)$ est "formellement" le cobord de la fonction $1 / 2 \tan \left(\frac{r-s}{2}\right)$, qui n'est pas dans $\mathcal{L}_{\mathbb{R}}^{2, \Delta}\left(\mathrm{S}^{1} \times \mathrm{S}^{1}\right)$. Le 
fait important est que, si $\phi$ est une représentation à valeurs dans $\operatorname{Diff}_{+}^{1+\alpha}\left(\mathrm{S}^{1}\right)$, avec $\alpha>1 / 2$, alors $\mathcal{L}_{\mathbb{R}}^{2, \Delta}\left(\mathrm{S}^{1} \times \mathrm{S}^{1}\right)$, comme il est démontré dans la proposition suivante. Ainsi, $c$ est un vrai cocycle à valeurs dans $\mathcal{L}_{\mathbb{R}}^{2, \Delta}\left(\mathrm{S}^{1} \times \mathrm{S}^{1}\right)$.

Proposition 2.1 (Segal-Reznikov). - Si $\phi: \Gamma \rightarrow \operatorname{Diff}_{+}^{1+\alpha}\left(\mathrm{S}^{1}\right), \alpha>1 / 2$, alors $c(g)$ appartient à $\mathcal{L}_{\mathbb{R}}^{2, \Delta}\left(\mathrm{S}^{1} \times \mathrm{S}^{1}\right)$ pour tout $g \in \Gamma$.

Démonstration. - On note d'abord qu'il existe une fonction continue et antisymétrique $K_{1}: \mathbb{R} \times \mathbb{R} \rightarrow \mathbb{R}$ périodique de période $2 \pi$ en chaque variable, et telle que pour tous $r, s \in[0,2 \pi]$ on a

$$
\frac{1}{\tan \left(\frac{r-s}{2}\right)}=2\left[\frac{1}{r-s}+K_{1}(r, s)\right] .
$$

Donc, pour montrer que $c(g) \in \mathcal{L}_{\mathbb{R}}^{2, \Delta}\left(\mathrm{S}^{1} \times \mathrm{S}^{1}\right)$ on doit montrer que

$$
\left[\frac{\left[g^{\prime}(r) g^{\prime}(s)\right]^{1 / 2}}{g(r)-g(s)}-\frac{1}{r-s}\right] \in \mathcal{L}_{\mathbb{R}}^{2, \Delta}\left(\mathrm{S}^{1} \times \mathrm{S}^{1}\right) .
$$

Pour $r, s \in \mathrm{S}^{1}$ tels que $|r-s|<\pi$, il existe un point $t$ dans le segment le plus court de $\mathrm{S}^{1}$ joignant ces deux points tel que $|g(r)-g(s)|=g^{\prime}(t)|r-s|$. On obtient alors

$$
\begin{aligned}
\left|\frac{\left[g^{\prime}(r) g^{\prime}(s)\right]^{1 / 2}}{g(r)-g(s)}-\frac{1}{r-s}\right| & =\frac{1}{|r-s| g^{\prime}(t)} \cdot\left|\left[g^{\prime}(r) g^{\prime}(s)\right]^{1 / 2}-g^{\prime}(t)\right| \\
& =\frac{1}{|r-s| g^{\prime}(t)} \cdot \frac{\left|g^{\prime}(r) g^{\prime}(s)-g^{\prime}(t)^{2}\right|}{\left[g^{\prime}(r) g^{\prime}(s)\right]^{1 / 2}+g^{\prime}(t)} . \\
& \leqslant \frac{1}{2 \inf \left(g^{\prime}\right)^{2}|r-s|}\left[\left|g^{\prime}(r)-g^{\prime}(t)\right| g^{\prime}(s)+g^{\prime}(t)\left|g^{\prime}(s)-g^{\prime}(t)\right|\right] .
\end{aligned}
$$

Comme $g^{\prime}$ est $\alpha$-Hölder continue, on obtient

$$
\left|\frac{\left[g^{\prime}(r) g^{\prime}(s)\right]^{1 / 2}}{g(r)-g(s)}-\frac{1}{r-s}\right| \leqslant \frac{\left|g^{\prime}\right|_{\alpha} \sup \left(g^{\prime}\right)}{2|r-s| \inf \left(g^{\prime}\right)^{2}}\left[|r-t|^{\alpha}+|s-t|^{\alpha}\right] \leqslant C|r-s|^{\alpha-1} .
$$

Puisque $\alpha>1 / 2$, la fonction $(r, s) \mapsto|r-s|^{\alpha-1}$ appartient à $\mathcal{L}_{\mathbb{R}}^{2}\left(\mathrm{~S}^{1} \times \mathrm{S}^{1}\right)$, et ceci montre que $c(g) \in \mathcal{L}_{\mathbb{R}}^{2, \Delta}\left(\mathrm{S}^{1} \times \mathrm{S}^{1}\right)$.

Remarque 2.2. - C'est la seule partie de la preuve du théorème où on utilise l'hypothèse $\alpha>1 / 2$.

Remarque 2.3. - Le cocycle que nous avons considéré n'apparaît pas de façon explicite dans [16] ou [17]. Dans ces travaux, la représentation est considérée sur l'espace des opérateurs de Hilbert-Schmidt de $\mathcal{L}^{2}\left(\mathrm{~S}^{1}\right)$, et le cocycle est associé à une transformée de Hilbert. Nous avons réinterprété cette construction en remarquant simplement que l'espace des opérateurs de HilbertSchmidt considéré s'identifie à $\mathcal{L}^{2}\left(\mathrm{~S}^{1} \times \mathrm{S}^{1}\right)$.

\subsection{Le courant géodésique}

Soit $\mathbb{D}$ le disque de Poincaré. Nous identifions le bord de $\mathbb{D}$ au cercle $\mathrm{S}^{1}$. Un courant géodésique est une mesure de Radon définie sur l'espace des géodésiques non orientées de $\mathbb{D}$. Notons que chaque géodésique non orientée est uniquement déterminée par ses extrémités sur le 
cercle, lesquelles sont des points distincts. Ainsi, un courant géodésique est une mesure positive $\mu$ définie pour les boréliens de $\mathrm{S}^{1} \times \mathrm{S}^{1}$ disjoints de la diagonale $\Delta$, qui est finie sur les ensembles compacts de $\mathrm{S}^{1} \times \mathrm{S}^{1}-\Delta$, et qui vérifie la condition de symétrie

$$
\mu([a, b] \times[c, d])=\mu([c, d] \times[a, b]), \quad a<b<c<d<a .
$$

On dit qu'un homéomorphisme $g$ du cercle préserve le courant $\mu$ si on a

$$
\mu([g(a), g(b)] \times[g(c), g(d)])=\mu([a, b] \times[c, d])
$$

pour tout $a<b<c<d<a$.

Exemple 2.4. - La mesure

$$
\mu^{L v}:=\frac{\mathrm{d} r \mathrm{~d} s}{4 \sin ^{2}\left(\frac{r-s}{2}\right)}
$$

définit un courant géodésique, lequel est invariant par l'action de $\operatorname{PSL}(2, \mathbb{R})$. En effet, pour ce courant on a $\mu^{L v}([a, b] \times[c, d])=\log \left[\mathrm{e}^{\mathrm{i} a}, \mathrm{e}^{\mathrm{i} b}, \mathrm{e}^{\mathrm{i} c}, \mathrm{e}^{\mathrm{i} d}\right]$, où $[\cdot, \cdot, \cdot, \cdot]$ dénote le birapport (voir [2]). Cette mesure est appelée la mesure de Liouville.

Reprenons maintenant la représentation et le cocycle du $\$ 2.1$, toujours sous l'hypothèse $\alpha>1 / 2$. Notons que, $\Gamma$ étant discret, cette représentation et son cocycle associé sont automatiquement continus. Donc, si $\Gamma$ possède la propriété de Kazhdan, alors le cocycle (1) est un cobord. En d'autres termes, il existe $K \in \mathcal{L}_{\mathbb{R}}^{2, \Delta}\left(\mathrm{S}^{1} \times \mathrm{S}^{1}\right)$ tel que pour tout $g \in \Gamma$ on a $(r, s)$-presque-partout :

$$
\frac{\left[g^{\prime}(r) g^{\prime}(s)\right]^{1 / 2}}{2 \tan \left(\frac{g(r)-g(s)}{2}\right)}-\frac{1}{2 \tan \left(\frac{r-s}{2}\right)}=K(g(r), g(s))\left[g^{\prime}(r) g^{\prime}(s)\right]^{1 / 2}-K(r, s),
$$

c'est-à-dire

$$
\left[\frac{1}{2 \tan \left(\frac{g(r)-g(s)}{2}\right)}-K(g(r), g(s))\right]^{2} g^{\prime}(r) g^{\prime}(s)=\left[\frac{1}{2 \tan \left(\frac{r-s}{2}\right)}-K(r, s)\right]^{2} .
$$

On a donc la proposition suivante.

Proposition 2.5. - Soit $\phi: \Gamma \rightarrow \operatorname{Diff}_{+}^{1+\alpha}\left(\mathrm{S}^{1}\right)$ une représentation, avec $\alpha>1 / 2$. Si $\Gamma$ a la propriété $(\mathrm{T})$ alors il existe un courant géodésique invariant par $\Gamma$ de la forme

$$
\mu_{K}=\left[\frac{1}{2 \tan \left(\frac{r-s}{2}\right)}-K(r, s)\right]^{2} \mathrm{~d} r \mathrm{~d} s,
$$

où $K \in \mathcal{L}_{\mathbb{R}}^{2, \Delta}\left(\mathrm{S}^{1} \times \mathrm{S}^{1}\right)$.

Remarque 2.6. - La condition $K(x, y)+K(y, x)=0$ imposée dans le $\$ 2.2$ est naturelle, parce qu'on veut que la mesure $\mu_{K}$ soit symétrique (et donc un courant géodésique). Néanmoins, cette restriction n'est pas très sérieuse, parce qu'on peut toujours remplacer une mesure $\mu$ par sa symétrisée $\bar{\mu}$ définie par $\bar{\mu}([a, b] \times[c, d]):=\mu([a, b] \times[c, d])+\mu([c, d] \times[a, b])$.

Puisque $\mu_{K}$ est absolument continue par rapport à la mesure de Lebesgue sur $\mathrm{S}^{1} \times \mathrm{S}^{1}-\Delta$, la propriété suivante est évidente :

$$
\mu_{K}([a, a] \times[b, c])=0, \quad a<b \leqslant c<a .
$$


D'autre part, nous verrons que le fait que $K$ soit une fonction de carré integrable entraîne une autre propriété pour $\mu_{K}$ :

$$
\mu_{K}([a, b) \times(b, c])=+\infty, \quad a<b<c<a .
$$

Pour démontrer (4) on note que

$$
\begin{gathered}
\mu_{K}([a, x] \times[y, c])^{1 / 2}=\left(\int_{a}^{x} \int_{y}^{c}\left[\frac{1}{2 \tan \left(\frac{r-s}{2}\right)}-K(r, s)\right]^{2} \mathrm{~d} r \mathrm{~d} s\right)^{1 / 2} \\
\geqslant\left(\int_{a}^{x} \int_{y}^{c} \frac{\mathrm{d} r \mathrm{~d} s}{4 \tan ^{2}\left(\frac{r-s}{2}\right)}\right)^{1 / 2}-\|K\|_{2}, \\
\int_{a}^{x} \int_{y}^{c} \frac{\mathrm{d} r \mathrm{~d} s}{4 \tan ^{2}\left(\frac{r-s}{2}\right)}=\int_{a}^{x} \int_{y}^{c} \frac{1}{4}\left[\frac{1}{\sin ^{2}\left(\frac{r-s}{2}\right)}-1\right] \mathrm{d} r \mathrm{~d} s \geqslant \log \left[\mathrm{e}^{\mathrm{i} a}, \mathrm{e}^{\mathrm{i} x}, \mathrm{e}^{\mathrm{i} y}, \mathrm{e}^{\mathrm{i} c}\right]-\pi^{2} .
\end{gathered}
$$

Puisque $\left[\mathrm{e}^{\mathrm{i} a}, \mathrm{e}^{\mathrm{i} x}, \mathrm{e}^{\mathrm{i} y}, \mathrm{e}^{\mathrm{i} c}\right]$ tend vers $+\infty$ quand $x$ et $y$ tendent vers $b$ (avec $a<x<b<y<c$ ), on obtient l'égalité (4).

En général, nous dirons qu'un courant géodésique est stable s'il vérifie les propriétés (3) et (4). Nous avons donc la proposition suivante.

Proposition 2.7. - Soit $\phi: \Gamma \rightarrow \operatorname{Diff}_{+}^{1+\alpha}\left(\mathrm{S}^{1}\right)$ une représentation, avec $\alpha>1 / 2$. Si $\Gamma$ a la propriété $(\mathrm{T})$ alors il existe un courant géodésique stable invariant par $\Gamma$.

\section{Fin de la preuve}

Les propriétés de stabilité du courant géodésique $\mu_{K}$ entraînent une certaine rigidité pour les applications qui préservent ce courant. Plus précisément, on a la proposition suivante.

Proposition 3.1. - Soit $f$ un homéomorphisme direct du cercle qui préserve un courant stable $\mu$. Si f fixe trois points alors $f$ est égal à l'identité.

Démonstration. - Soit $I=(a, b)$ une composante connexe du complémentaire de l'ensemble de points fixes de $f$. Remarquons que $a$ et $b$ sont des points fixes de $f$. Soit $c \in(b, a)$ un autre point fixe de $f$.

Soit $x$ un point arbitraire de $(a, b)$. Puisque $f$ n'a pas de points fixes dans $(a, b)$, quitte à changer $f$ par $f^{-1}$, on peut supposer que $\lim _{i \rightarrow+\infty} f^{i}(x)=b$, et $\operatorname{donc}_{\lim _{i \rightarrow-\infty}} f^{i}(x)=a$. On a alors

$$
\mu([a, x] \times[b, c])=\mu([a, f(x)] \times[b, c]),
$$

et donc $\mu([x, f(x)] \times[b, c])=0$. En général, pour tout $i \in \mathbb{Z}$ on a

$$
\mu\left(\left[f^{i}(x), f^{i+1}(x)\right] \times[b, c]\right)=0 .
$$

On conclut que

$$
\mu((a, b) \times[b, c])=\sum_{i \in \mathbb{Z}} \mu\left(\left[f^{i}(x), f^{i+1}(x)\right] \times[b, c]\right)=0,
$$


ce qui contredit (4).

Grâce au lemme précédent et à la proposition (2.7), on obtient le lemme suivant (nous reprenons la notation $\phi(g)$ au lieu de $g^{-1}$ pour des raisons qui seront bientôt claires).

LEMME 3.2. - Soit $\phi: \Gamma \rightarrow \operatorname{Diff}_{+}^{1+\alpha}\left(\mathrm{S}^{1}\right)$ une représentation, avec $\alpha>1 / 2$. Si $\Gamma$ a la propriété $(\mathrm{T})$ et $g \in \Gamma$ est tel que $\phi(g)$ fixe trois points du cercle, alors $\phi(g)$ est l'identité.

Le lemme suivant, dû à D. Witte, permet de montrer que, sous nos hypothèses, l'action de $\phi(\Gamma)$ sur $\mathrm{S}^{1}$ est libre.

LEMME 3.3. - Soit $\phi: \Gamma \rightarrow \operatorname{Diff}_{+}^{1+\alpha}\left(\mathrm{S}^{1}\right)$ une représentation, avec $\alpha>1 / 2$. Si $\Gamma$ a la propriété (T) et $g \in \Gamma$ est tel que $\phi(g)$ fixe un point du cercle, alors $\phi(g)$ est l'identité.

Démonstration. - On considère le revêtement à trois feuillets du cercle, que l'on note $\widehat{\mathrm{S}}^{1}$. Sur ce revêtement agit (par difféomorphismes de classe $C^{1+\alpha}$ ) une extension $\widehat{\Gamma}$ de $\Gamma$ de la forme

$$
0 \longrightarrow \mathbb{Z} / 3 \mathbb{Z} \longrightarrow \widehat{\Gamma} \longrightarrow \Gamma \longrightarrow 0 .
$$

Puisque $\Gamma$ a la propriété $(\mathrm{T})$ et $\mathbb{Z} / 3 \mathbb{Z}$ est fini, $\widehat{\Gamma}$ vérifie encore la propriété (T) (voir [14], p. 9). Si $g \in \Gamma$ est tel que $\phi(g)$ fixe un point du cercle initial, alors l'une de ses préimages dans $\widehat{\Gamma}$ fixe trois points de $\widehat{\mathrm{S}}^{1}$ par l'action induite. Comme $\widehat{\mathrm{S}}^{1}$ s'identifie au cercle, on en déduit, d'après le lemme précédent, que $\phi(g)$ est égal à l'identité.

On peut maintenant finir la preuve de notre théorème. En effet, par le lemme précédent, le groupe $\phi(\Gamma)$ agit librement sur le cercle. Par un ancien théorème de Hölder (voir [12] ou [9]), le groupe $\phi(\Gamma)$ est abélien. Puisque $\phi(\Gamma)$ vérifie encore la propriété (T), il doit être fini (voir le Chapitre 1 de [14]).

\section{Le cas général}

Notre théorème s'étend de manière presque directe au cas des groupes localement compacts non discrets ayant la propriété $(\mathrm{T})$. On obtient ainsi le théorème suivant.

THÉORÈME 4.1. - Soit $\Gamma$ un groupe topologique localement compact, séparable et à base dénombrable, et soit $\phi: \Gamma \rightarrow \operatorname{Diff}_{+}^{1+\alpha}\left(\mathrm{S}^{1}\right), \alpha>1 / 2$, une représentation continue par rapport à la topologie $\mathrm{C}^{1+\alpha}$. Si $\Gamma$ possède la propriété $(\mathrm{T})$, alors l'image $\phi(\Gamma)$ est topologiquement conjuguée à un sous-groupe compact de $\mathrm{SO}(2, \mathbb{R})$.

Démonstration. - D'abord, il faut vérifier la continuité de la représentation $\theta$ et du cocycle $c$ du $\S 2.1$. Notons $i d$ l'élément neutre de $\Gamma$. Pour démontrer que la représentation $\theta$ est continue, chaque $\theta(g)$ étant unitaire, il suffit de vérifier la continuité en $i d \in \Gamma$ de l'application $g \mapsto \theta(g) K$ pour chaque fonction $K$ continue. Nous laissons au lecteur le soin de vérifier que cette continuité résulte immédiatement du théorème de convergence dominée.

Pour démontrer que l'application $g \mapsto c(g)$ est continue, il suffit de vérifier la continuité en $i d \in \Gamma$. De façon analogue à la preuve du théorème (2.1), on montre que

$$
\left|\frac{\left[g^{\prime}(r) g^{\prime}(s)\right]^{1 / 2}}{g(r)-g(s)}-\frac{1}{s-r}\right| \leqslant \frac{\left|g^{\prime}\right|_{\alpha} \sup \left(g^{\prime}\right)}{\inf \left(g^{\prime}\right)^{2}} \cdot|r-s|^{\alpha-1} .
$$


En notant $C=\int_{\mathrm{S}^{1}} \int_{\mathrm{S}^{1}}|r-s|^{2(\alpha-1)} \mathrm{d} r \mathrm{~d} s<\infty$, on obtient

$$
\int_{\mathrm{S}^{1}} \int_{\mathrm{S}^{1}}\left|\frac{\left[g^{\prime}(r) g^{\prime}(s)\right]^{1 / 2}}{g(s)-g(r)}-\frac{1}{s-r}\right|^{2} \mathrm{~d} r \mathrm{~d} s \leqslant C \frac{\left|g^{\prime}\right|_{\alpha} \sup \left(g^{\prime}\right)}{\inf \left(g^{\prime}\right)^{2}} .
$$

Puisque la représentation $\phi$ est continue, cette dernière quantité est petite pour $g$ proche de $i d$ dans $\Gamma$.

À partir d'ici on peut suivre les arguments de la démonstration du cas discret, à ceci près que, dans le cas présent, on conclut seulement que $\phi(\Gamma)$ est compact. Puisque tout sous-groupe compact de Homéo $+\left(\mathrm{S}^{1}\right)$ est topologiquement conjugué à un groupe de rotations (voir [9]), ceci achève la preuve du théorème.

Ce dernier théorème est probablement vrai pour les représentations continues dans Homéo $_{+}\left(\mathrm{S}^{1}\right)$ des groupes non discrets localement compacts à base dénombrable. En effet, si $\Gamma$ est localement compact et $\phi: \Gamma \rightarrow$ Homéo $_{+}\left(\mathrm{S}^{1}\right)$ est une représentation continue, alors une application du théorème de Montgomery-Zippin montre que la composante connexe de $I d$ dans $\phi(\Gamma)$, munie de la topologie induite de $\Gamma$ par $\phi$, est un groupe de Lie. D'autre part, la classification des actions fidèles des groupes de Lie connexes par homéomorphismes directs du cercle est connue (voir [8] et [9]). On sait que ces actions transitent par des homomorphismes de $\Gamma$ sur $(\mathbb{R},+), \operatorname{SO}(2, \mathbb{R}), \operatorname{PSL}(2, \mathbb{R}), \mathrm{PSL}_{k}(2, \mathbb{R})$ ou $\widetilde{\mathrm{SL}}(2, \mathbb{R})$. Il est alors facile de démontrer que si $\Gamma$ est connexe et possède la propriété $(\mathrm{T})$, alors l'image $\phi(\Gamma)$ est triviale ou conjuguée à $\mathrm{SO}(2, \mathbb{R})$.

Néanmoins, si $\Gamma$ est un groupe localement compact non connexe et non discret ayant la propriété (T), alors la situation est plus compliquée. Ceci est dû au fait que la composante connexe d'un groupe de Lie ayant la propriété $(\mathrm{T})$ ne possède pas nécessairement cette propriété, comme le montre l'exemple $\mathbb{R}^{3} \rtimes \mathrm{SL}(3, \mathbb{Z})$. Pourtant, il est raisonnable de penser que $\phi(\Gamma)$ est encore conjuguée à un sous-groupe compact de $\mathrm{SO}(2, \mathbb{R})$.

\section{Une autre preuve du théorème}

Puisque l'annulation du cocycle (1) entraîne l'existence d'un courant géodésique stable invariant par le groupe, il est naturel se demander quel est le groupe des homéomorphismes directs du cercle qui préservent ce courant. Dans cette direction, on a le résultat suivant.

PROPOSITION 5.1. - Si $\mu$ est un courant géodésique stable quelconque, alors le groupe des homéomorphismes directs du cercle qui préservent $\mu$ est topologiquement conjugué à un sousgroupe de $\operatorname{PSL}(2, \mathbb{R})$.

L'idée de la preuve est de montrer que, sous ces hypothèses, le groupe en considération vérifie la propriété de convergence, et on peut donc appliquer le théorème de Tukia, Hinkkanen, CassonJungreis et Gabai (voir $[18,13,5,7]$ respectivement). Pour les détails, nous renvoyons le lecteur à [15].

Remarque 5.2. - La proposition (5.1) ne signifie pas en général que le groupe des homéomorphismes directs de $S^{1}$ qui préservent $\mu$ est topologiquement conjugué à $\operatorname{PSL}(2, \mathbb{R})$ tout entier par un homéomorphisme qui transforme le courant $\mu$ dans la mesure de Liouville. En fait, dans [2] il est démontré que ceci a lieu si et seulement si $\mu$ satisfait l'égalité

$$
\mathrm{e}^{-\mu([a, b] \times[c, d])}+\mathrm{e}^{-\mu([b, c] \times[d, a])}=1
$$

pour tout $a<b<c<d<a$. 
Remarque 5.3. - Il est intéressant aussi de signaler que si un courant $\mu$ s'exprime sous la forme $\mu=\mu_{K}$, avec $K \in \mathcal{L}_{\mathbb{R}}^{2, \Delta}\left(\mathrm{S}^{1} \times \mathrm{S}^{1}\right)$, et si le groupe des homéomorphismes qui préservent $\mu$ n'est pas compact, alors la conjugaison donnée par la proposition (5.1) ne peut pas être de classe $C^{1+\alpha}, \alpha>1 / 2$. Ceci est une conséquence du travail de A. Reznikov (voir [17]).

Puisque les seuls sous-groupes de $\operatorname{PSL}(2, \mathbb{R})$ qui ont la propriété $(\mathrm{T})$ sont les groupes compacts (voir [14], p. 80), la proposition (5.1) permet de donner une autre preuve de notre théorème.

\section{Remerciements}

Je remercie Étienne Ghys pour m'avoir proposé ce problème et m'avoir signalé l'existence de [17], ainsi que pour son soutien constant dans ce travail. Je remercie vivement aussi D. Witte pour m'avoir indiqué l'argument essentiel du §3 qui permet d'établir directement notre résultat sans faire référence au théorème de convergence. Finalement, je remercie T. Barbot, F. Beguin, R. Feres, P. de la Harpe, A. Guillot et A. Zuk pour leur intérêt, leurs remarques et corrections.

Je voudrais dédier ce travail à la mémoire de Carlos Cid A.

\section{RÉFÉRENCES}

[1] ALPERIN R., Locally compact groups acting on trees and property (T), Mh. Math. 93 (1982) 261-265.

[2] Bonahon F., The geometry of Teichmüller space via geodesic currents, Inv. Math. 92 (1988) 139162.

[3] Burger M., Monod N., Bounded cohomology of lattices in higher rank Lie groups, J. Eur. Math. Soc. (JEMS) 1 (1999) 199-235.

[4] Burger M., Monod N., Continuous bounded cohomology and applications to rigidity theory. Geometric and Functional Analysis, à paraître.

[5] Casson A., Jungreis D., Convergence groups and Seifert fibered 3-manifolds, Inv. Math. 118 (1994) 441-456.

[6] FARB B., Shalen P., Real-analytic actions of lattices, Inv. Math. 135 (1999) 273-296.

[7] GABAi D., Convergence groups are Fuchsian groups, Ann. of Math. 136 (1992) 447-510.

[8] GHys É., Actions de réseaux sur le cercle, Inv. Math. 137 (1999) 199-231.

[9] GHYs É., Groups acting on the circle, L'Enseignement Mathématique, à paraître.

[10] Gromov M., Hyperbolic groups, in : Essays in Group Theory, Springer-Verlag, 1987, pp. 75-263.

[11] Ghys É., Sergiescu V., Sur un groupe remarquable de difféomorphismes du cercle, Comment. Math. Helvetici 62 (1987) 185-239.

[12] Hector G., Hirsch U., Introduction to the Geometry of Foliations. Part B. Foliations of Codimension One, Second Edition, in: Aspect of Mathematics, 1987.

[13] HinkKanen A., Abelian and nondiscrete convergence groups of the circle, Trans. AMS 318 (1990) $87-121$.

[14] De la HaRpe P., VALetTe A., La propriété (T) de Kazhdan pour les groupes localement compacts, Astérisque 175 (1989).

[15] NaVAs A., Actions de groupes de Kazhdan sur le cercle, Prépublication de l'ENS, Lyon.

[16] Pressley A., Segal G., Loop Groups, in : Oxford Mathematical Monographs, 1986.

[17] ReZnikov A., Analytic topology of groups, actions, strings and varieties. Chapter $2:$ A theory of groups acting in the circle. Preprint, pp. 59-65 (August 11, 1999). http://xxx.lpthe.jussieu.fr/find/ math/1/au:+Reznikov_A/0/1/0/all/0/1.

[18] TUKIA P., Homeomorphic conjugates of Fuchsian groups, J. Reine Angew. Math. 391 (1988) 1-54.

[19] Watatani Y., Property (T) of Kazhdan implies property (FA) of Serre, Math. Japon 27 (1981) 97 103. 
[20] WitTe D., Arithmetic groups of higher $\mathbb{Q}$-rank cannot act on 1-manifolds, Proc. AMS 122 (1994) 333-340.

[21] WitTe D., Products of similar matrices, Proc. AMS 126 (1998) 1005-1015.

[22] ZuK A., Property (T) and Kazhdan constants for discrete groups, Prépublication de l'ENS, Lyon.

(Manuscrit reçu le 17 septembre 2001 ; accepté, après révision, le 12 février 2002.)

Andrés NAVAS

Unité de Mathématiques Pures et Appliquées,

École Normale Supérieure de Lyon,

UMR 5669 du CNRS,

46 allée d'Italie,

F-69364 Lyon 07, France

E-mail : anavas@umpa.ens-lyon.fr 